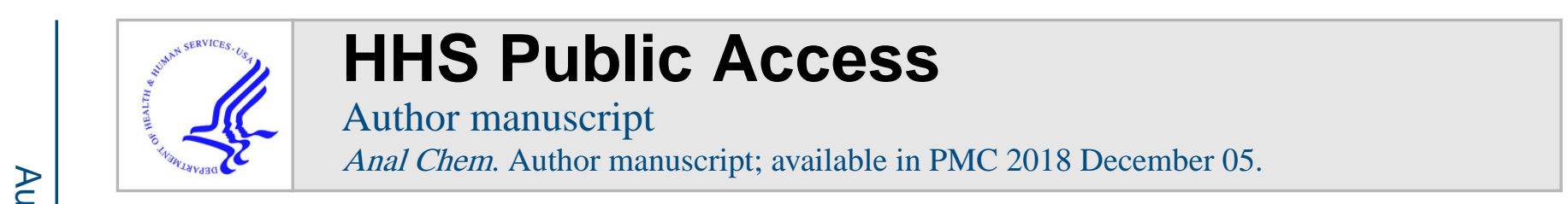

Published in final edited form as:

Anal Chem. 2017 December 05; 89(23): 12626-12630. doi:10.1021/acs.analchem.7b03967.

\title{
Plasmonic Sensing with 3D Printed Optics
}

\author{
Samuel S. Hinman ${ }^{\dagger}$, Kristy S. McKeating ${ }^{\ddagger}$, and Quan Cheng ${ }^{\star}, \dagger, \ddagger$ \\ †Environmental Toxicology, University of California-Riverside, Riverside, California 92521, United \\ States \\ ¥Department of Chemistry, University of California-Riverside, Riverside, California 92521, United \\ States
}

\begin{abstract}
Three-dimensional (3D) printing has undergone an exponential growth in popularity due to its revolutionary and near limitless manufacturing capabilities. Recent trends have seen this technology utilized across a variety of scientific disciplines, including the measurement sciences, but precise fabrication of optical components for high-performance biosensing has not yet been demonstrated. We report here 3D printing of high-quality, custom prisms by stereolithography that enable Kretschmann-configured plasmonic sensing of bacterial toxins. Simple benchtop polishing procedures render a smooth surface that supports propagation of surface plasmon polaritons with a deposited gold layer, which exhibit high bulk refractive index sensitivities and are capable of discriminating trace levels of cholera toxin on a supported lipid membrane interface. Further evidence of the flexibility of this manufacturing technique is demonstrated with printed prisms of varied geometries and in situ monitoring of nanoparticle growth by total internal reflection spectroscopy. This work represents the first example of 3D printed light-guiding sensing platforms and demonstrates the versatility and broad perspective of 3D printing in optical detection.
\end{abstract}

\section{Graphical abstract}

\footnotetext{
“Corresponding Author: Phone: (951) 827-2702. Fax: (951) 827-4713. quan.cheng@ucr.edu.

Supporting Information

The Supporting Information is available free of charge on the ACS Publications website at DOI: 10.1021/acs.analchem.7b03967. Experimental details, alternative prism treatments (Figure S1), Sellmeier fitting of ellipsometry data (Figure S2), SPR reflectivity curves (Figure S3), and dove prism absorbance measurements (Figure S4) (PDF)

ORCID

Samuel S. Hinman: 0000-0002-7727-0450

Kristy S. McKeating: 0000-0001-7281-1804

Quan Cheng: 0000-0003-0934-358X

Notes

The authors declare no competing financial interest.
} 


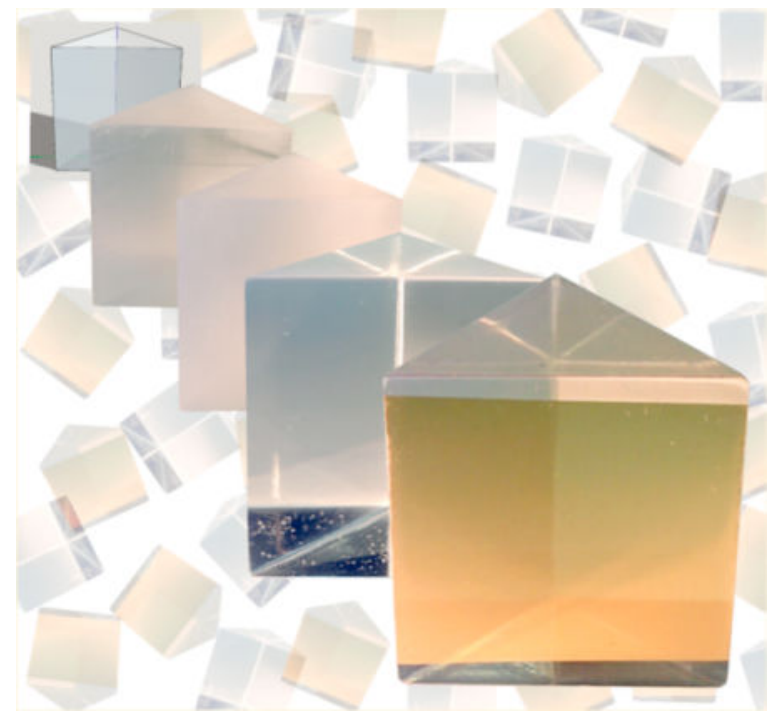

Three-dimensional (3D) printing has revolutionized material production in many facets of society, from industry to consumer levels, as the technology enables the rapid translation of original ideas into physical objects, with minimal training or expertise required on behalf of the end user. In the analytical field, 3D printing techniques have largely been exploited for the prototyping of fluidic devices and custom mechanical parts, ${ }^{1,2}$ with the acts of guiding and manipulating light through 3D printed optics evading investigation. Sample handling has remained a common motif in analytical 3D printing, with the manufacturing of silica gel patterns for chromatography, ${ }^{3}$ solid phase extraction devices,,${ }^{4,5}$ microfluidic sample preconcentrators, ${ }^{6}$ and microchannel chips ${ }^{7-10}$ previously demonstrated. However, there have been limited attempts to fabricate integrated biosensors and biorecognition elements into 3D printed devices, ${ }^{11}$ with the majority of signal transduction being electrochemical. $10,12,13$

Within this letter, a stereolithographic approach for the manufacturing of optical components, notably high-quality, custom prisms for biosensing, is presented. Direct 3D printing of equilateral prisms leads to variable surface roughness. By applying simple benchtop polishing procedures we generated the photoplastic surface amenable to optical techniques that require high precision, which we then demonstrated their use for surface plasmon resonance (SPR) sensing (Figure 1A). 3D printed equilateral prisms were applied in SPR assays with a nanoscale layer of gold deposited on one face, which exhibited high bulk refractive index sensitivities, comparable to commercial high refractive index glasses. Further versatility is demonstrated with 3D printed dove prisms, on which in situ nanoparticle growth and label-free monitoring were successfully performed. This approach not only allows for versatile optics with individually dictatable geometry but also renders the fabrication of optical components, typically restricted to specialized manufacturers of high refractive index glasses, accessible to a larger community.

A commercial, clear photoactive resin was used for prism fabrication by 3D printing, which consists of a proprietary mixture of methacrylated oligomers, a methacrylated monomer, and 
photoinitiator(s). ${ }^{14}$ Once cured, the material resembles poly(methyl methacrylate) (PMMA) and is relatively transparent (Figure 1A). However, direct printing of the equilateral prisms resulted in a highly variable surface roughness ( $\mathrm{rms}=326 \mathrm{~nm}, 20 \mu \mathrm{m} \times 20 \mu \mathrm{m}$ areas, $n=3$ ), causing significant scattering of light passing through each face. Efforts were taken to render a smooth surface suitable for optical use. Among many methods tested (Figure S1, Supporting Information), a short (i.e., $30 \mathrm{~s}$ ) wet sanding of each face, followed by a benchtop polishing wheel treatment $(\sim 5 \mathrm{~min})$ proved highly effective for reducing the overall surface roughness $(\mathrm{rms}=23 \mathrm{~nm})$, which can be seen in the striking clarity of the material under naked eye inspection (Figure 1A), in addition to the decreased surface defects observed through scanning electron microscopy and atomic force microscopy (Figure 1B). For comparison, commercially available glasses exhibit surfaces roughness values of less than $2 \mathrm{~nm} \cdot{ }^{15,16}$ Once effectively treated, optical characterizations confirmed the similarity of the cured substrate to PMMA (Figure S2, Supporting Information), ${ }^{17,18}$ which were performed through ellipsometry and FT-IR spectroscopy (Figure 1C,D).

High-performance optical detection with the 3D printed prisms was demonstrated through surface plasmon resonance (SPR) assays. The basis of SPR is the surface plasmon polariton (SPP), which propagates along a planar, nanoscale gold surface in resonance with an incident light source of matching angular frequency. ${ }^{19,20}$ High refractive index prisms are typically used to increase the momentum of incident light and fulfill the resonant matching conditions, and must be smooth enough to allow SPP propagation parallel to the surface. Gold films $(50 \mathrm{~nm})$ were evaporated onto one face of the 3D printed prisms in the Kretschmann configuration, and their reflectivity images are provided in Figure 2A, with the microchannel flow cell used throughout these experiments clearly defined. The difference in contrast between the flow cell and background areas is due to the dielectric sensitive nature of SPR, as the flow cell medium(i.e., water) and channel defining material (i.e., PDMS) possess significantly different refractive indices. Plotting the reflected light intensity within the flow cell medium against the incident angle results in a characteristic SPR reflectivity curve (Figure $2 \mathrm{~B}$ ). The dip in reflectivity $\sim 82^{\circ}$ is indicative of SPP generation and optimal matching of the resonance conditions (e.g., incident wavelength and angle), which, under this experimental configuration, ${ }^{21}$ only occurs through a prism-mediated increase in the wavevector of incident light. Also notable in the spectrum is the critical angle $\sim 66^{\circ}$, at which light within the prism meets the conditions for total internal reflection. BK7 and SF2 prisms, typically used in commercial SPR instrumentation, show similar patterns though with reflectivity minima occurring at lower incident angles, which are the result of their higher indices of refraction (Figure S3, Supporting Information).

The refractive index sensitivity of 3D printed components was evaluated and compared to commercial glass prisms. Static injections of sodium chloride solutions possessing varying indices of refraction ( $n=1.333-1.350)$ over the gold film were used to establish sensor calibrations, and the reflectivity spectra for the printed substrates are provided in Figure 2B. With each increase in $\mathrm{NaCl}$ concentration, the SPR reflectivity dip shifts toward a higher incident angle and intensity. These injections can be monitored in real-time through either parameter, with a plot of time against $\Delta \% R$ (i.e., normalized intensity) provided in Figure 2C. When the bulk sensitivity was compared to gold films over BK7 and SF2 prisms, the 3D printed prisms exhibited a comparable sensitivity of $177.6^{\circ} \mathrm{RIU}^{-1}$ (Figure 2D). ${ }^{22}$ 
A biosensing interface for bacterial cholera toxin was constructed on the printed SPR surface using biomimetic lipid membranes. The prisms were immersed in a 3mercaptopropanol solution to form a self-assembled monolayer (SAM), rendering the gold surface hydrophilic and allowing for supported lipid membrane formation (Figure $3 \mathrm{~A}$ ). ${ }^{23}$ Utilization of supported membranes is beneficial as they allow the incorporation of specific recognition elements, including lipid receptors, while minimizing nonspecific binding from nontarget species due to the zwitterionic nature of phosphocholine headgroups. ${ }^{24}$ Preformed phosphocholine (POPC) vesicles incorporating $5 \%(\mathrm{n} / \mathrm{n}) \mathrm{GM}_{1}$ were flowed over the SAMcoated gold prisms to form a supported membrane (I in Figure 3A,B), and thereafter, cholera toxin $\left(0-20 \mu \mathrm{g} \mathrm{m}^{-1}\right)$ was introduced (II in Figure 3A-C). Each injection and incubation was followed by a $10 \mathrm{~min}$ rinse with phosphate buffer to remove any unbound molecules. Binding patterns for cholera toxin are strong and specific, with signal intensities comparable to established SPR sensors in the literature. ${ }^{25,26}$

An important advantage of 3D printing optical components is the ability to custom tailor designs to the task at hand. The use of dove prisms has been demonstrated in several SPR applications, which allow for the arrangement of optical components along a single axis, simplifying the instrumental setup. ${ }^{27}$ We further demonstrated the versatility of 3D printing by performing total internal reflection spectroscopic detection utilizing dove prisms with gold nanoparticles. In fact, gold nanoparticles have been utilized as an alternative layer of the evaporated gold films on these surfaces, circumventing the need for cleanroom facilities and vacuum equipment in sensor fabrication. ${ }^{28} 3 \mathrm{D}$ printing of dove prisms was carried out with the position of the incident light source tuned to allow for total internal reflection within the prism and its transmission to the spectrometer along the same axis (Figure 4A). Using an in situ polydopamine-mediated growth mechanism, ${ }^{29}$ gold nanoparticles were fabricated directly on the long face of each prism (Figure 4B,C) and the process was monitored in real time (Figure 4D). For each spectrum, background absorbance of a prism coated only with polydopamine was subtracted (Figure S4, Supporting Information). Consistent with standard localized surface plasmon resonance (LSPR) mechanisms, the nanoparticle absorbance peak increases in intensity throughout the $24 \mathrm{~h}$ period in which chloroauric acid was incubated at the surface, ${ }^{30}$ which remained constant after rinsing with nanopure water. This proof-of-concept experiment provides evidence that not only may prisms be fabricated on-demand through $3 \mathrm{D}$ printing, but full sensing materials may be constructed from them entirely using benchtop procedures, thus increasing sensor accessibility to a wide audience of researchers with varying technical backgrounds.

In conclusion, we have demonstrated for the first time the use of 3D printing technology to fabricate high-quality optical components for label-free biosensing. While the technology is still open to improvements, including higher resolutions and a broader range of optical characteristics, 3D printed optics offer flexibility that is unmatched by prefabricated units for verifying custom design and prototyping in sensor research. With prisms of varying geometry demonstrated here for SPR and LSPR, the construction of optical sensing materials by $3 \mathrm{D}$ printing has been rendered accessible to a large scientific audience. There are indeed instances where expensive, traditional glass materials are more desirable; researchers however can certainly benefit from the swift and low cost fabrication offered by 3D printing, especially for disposable, field deployable devices. With printer costs ever 
decreasing, we anticipate a wide adoption of the technique for 3D printed optical components, sensors, and light-guiding systems.

\section{Supplementary Material}

Refer to Web version on PubMed Central for supplementary material.

\section{Acknowledgments}

The authors gratefully acknowledge financial support from the National Science Foundation (Grant CHE-1413449). S.S.H. was supported by a University of California, Riverside (UCR) Dissertation Year Program (DYP) fellowship and an NIEHS T32 training grant (Grant T32 ES018827).

\section{References}

1. Zhang Y, Ge SG, Yu JH. TrAC, Trends Anal Chem. 2016; 85:166-180.

2. Gross B, Lockwood SY, Spence DM. Anal Chem. 2017; 89:57-70. [PubMed: 28105825]

3. Fichou D, Morlock GE. Anal Chem. 2017; 89:2116-2122. [PubMed: 28208299]

4. Kataoka EM, Murer RC, Santos JM, Carvalho RM, Eberlin MN, Augusto F, Poppi RJ, Gobbi AL, Hantao LW. Anal Chem. 2017; 89:3460-3467. [PubMed: 28230979]

5. Belka M, Ulenberg S, Baczek T. Anal Chem. 2017; 89:4373-4376. [PubMed: 28361532]

6. Su CK, Peng PJ, Sun YC. Anal Chem. 2015; 87:6945-6950. [PubMed: 26101898]

7. Au AK, Bhattacharjee N, Horowitz LF, Chang TC, Folch A. Lab Chip. 2015; 15:1934-1941. [PubMed: 25738695]

8. Chan HN, Shu YW, Xiong B, Chen YF, Chen Y, Tian Q, Michael SA, Shen B, Wu HK. ACS Sens. 2016; 1:227-234.

9. Tang CK, Vaze A, Rusling JF. Lab Chip. 2017; 17:484-489. [PubMed: 28067370]

10. Duarte LC, Chagas CLS, Ribeiro LEB, Coltro WKT. Sens Actuators, B. 2017; 251:427-432.

11. Mandon CA, Blum LJ, Marquette CA. Anal Chem. 2016; 88:10767-10772. [PubMed: 27723966]

12. Gowers SAN, Curto VF, Seneci CA, Wang C, Anastasova S, Vadgama P, Yang GZ, Boutelle MG. Anal Chem. 2015; 87:7763-7770. [PubMed: 26070023]

13. Loo AH, Chua CK, Pumera M. Analyst. 2017; 142:279-283. [PubMed: 28001145]

14. Clear Photoactive Resin for Form 1, Form 1+ and Form 2 Safety Data Sheet 2017 https:// formlabs.com/media/upload/Clear-SDS_u324bsC.pdf (accessed May 22, 2017)

15. Henke L, Nagy N, Krull UJ. Biosens Bioelectron. 2002; 17:547-555. [PubMed: 11959477]

16. Chada N, Sigdel KP, Gari RRS, Matin TR, Randall LL, King GM. Sci Rep. 2015; 5:12550. [PubMed: 26228793]

17. Sultanova N, Kasarova S, Nikolov I. Acta Phys Pol, A. 2009; 116:585-587.

18. Beadie G, Brindza M, Flynn RA, Rosenberg A, Shirk JS. Appl Opt. 2015; 54:F139-F143. [PubMed: 26560600]

19. Couture M, Zhao SS, Masson JF. Phys Chem Chem Phys. 2013; 15:11190-11216. [PubMed: 23748491]

20. Hinman SS, McKeating KS, Cheng Q. Anal Chem. 2017; doi: 10.1021/acs.analchem.7b04251

21. Kretschmann E, Raether H. Z Naturforsch, A: Phys Sci. 1968; 23:2135-2136.

22. Gupta G, Kondoh J. Sens Actuators, B. 2007; 122:381-388.

23. Wang ZZ, Wilkop T, Han JH, Dong Y, Linman MJ, Cheng Q. Anal Chem. 2008; 80:6397-6404. [PubMed: 18620431]

24. Li H, Dauphin-Ducharme P, Arroyo-Curras N, Tran CH, Vieira PA, Li S, Shin C, Somerson J, Kippin TE, Plaxco KW. Angew Chem, Int Ed. 2017; 56:7492-7495.

25. Hinman SS, Ruiz CJ, Drakakaki G, Wilkop TE, Cheng Q. ACS Appl Mater Interfaces. 2015; 7:17122-17130. [PubMed: 26193345] 
26. Hinman SS, McKeating KS, Cheng Q. Anal Chem. 2017; 89:4272-4279. [PubMed: 28316233]

27. Bolduc OR, Live LS, Masson JF. Talanta. 2009; 77:1680-1687. [PubMed: 19159783]

28. Yockell-Lelievre H, Lussier F, Masson JF. J Phys Chem C. 2015; 119:28577-28585.

29. Sureshkumar M, Lee PN, Lee CK. J Mater Chem. 2011; 21:12316-12320.

30. Willets KA, Van Duyne RP. Annu Rev Phys Chem. 2007; 58:267-297. [PubMed: 17067281] 
A

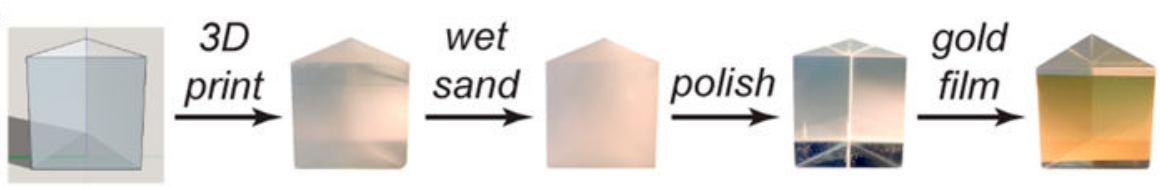

B

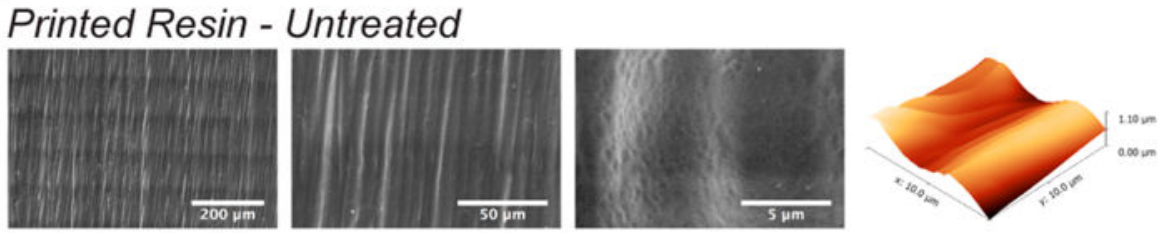

\section{Post Sanding and Polishing}
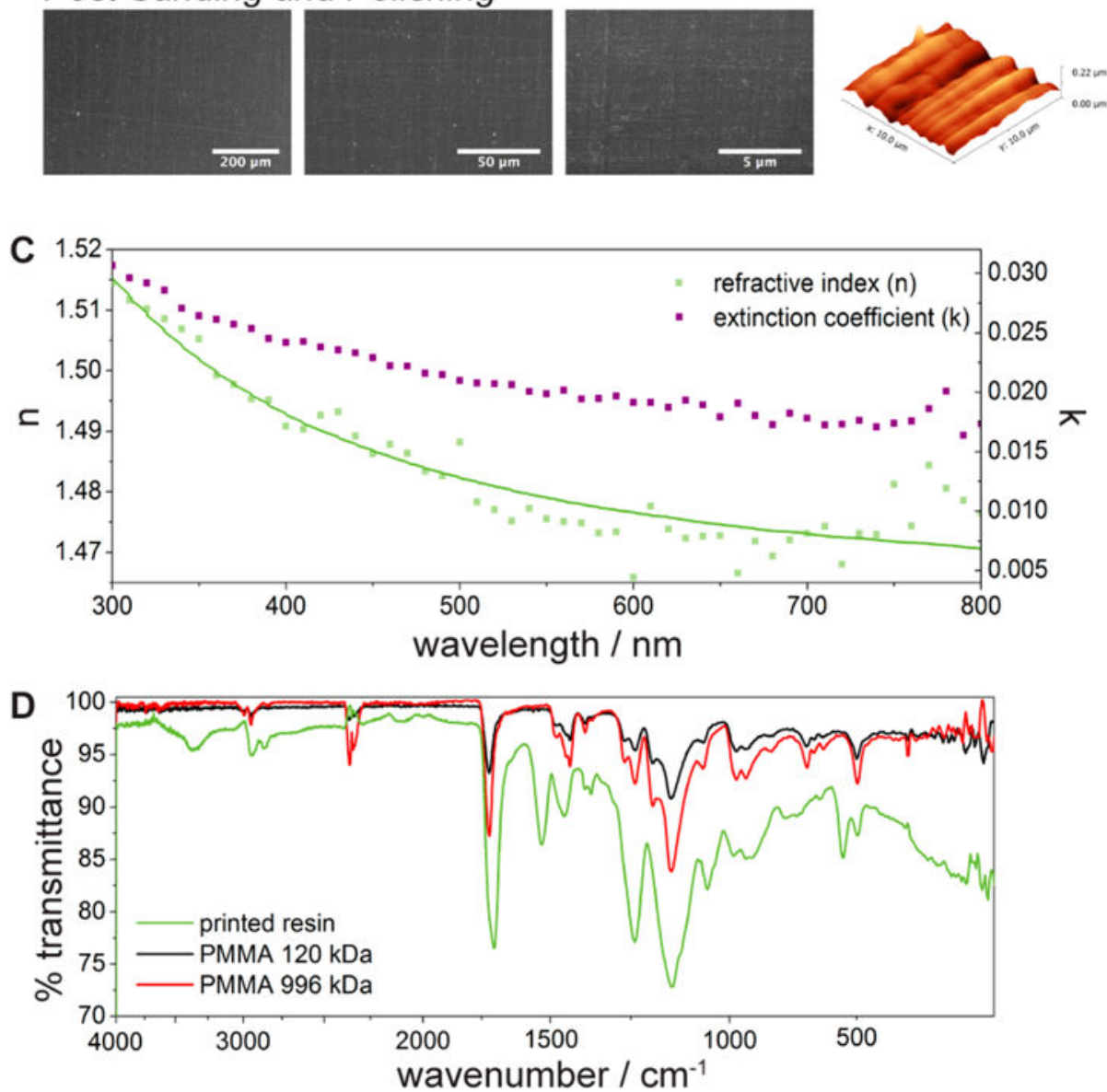

Figure 1.

Characterization of 3D printed optics. (A) Fabrication scheme for 3D printed surface plasmon resonance sensors. Each prism face measures $20 \mathrm{~mm} \times 20 \mathrm{~mm}$. (B) Scanning electron and atomic force microscopic characterizations of the surface before and after polishing treatment. (C) Ellipsometry results, depicting wavelength dependence of refractive index $(n)$ and extinction coefficient $(k)$ for the printed resin. Solid line depicts the Sellmeier fit for $n$. (D) FT-IR spectra of the cured resin and two different molecular weight PMMAs for standard comparisons. 
A
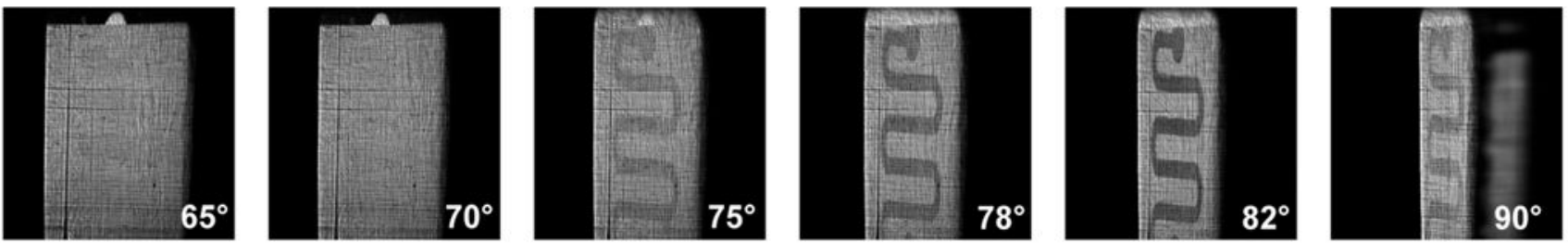

B

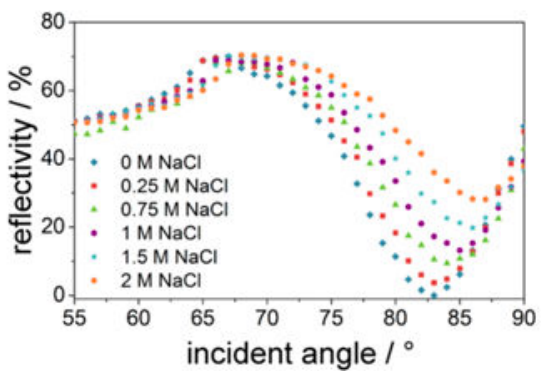

C

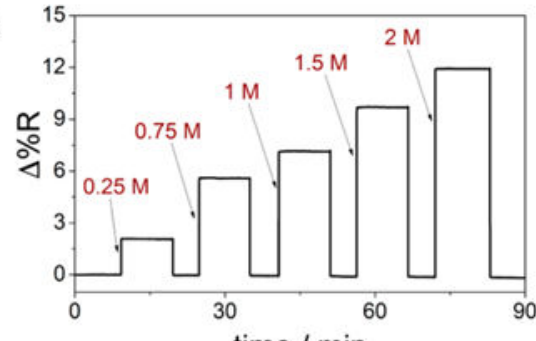

D

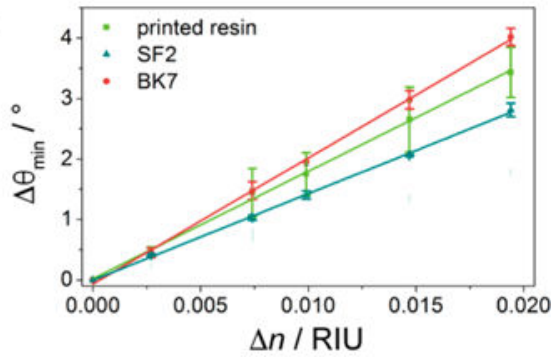

Figure 2.

3D printed surface plasmon resonance (SPR) sensors. (A) Reflected images depicting the dependence of plasmonic resonance at the gold-coated and 3D printed surface on incident light angle. (B) SPR reflectivity curves as the dielectric is varied from 0 to $2 \mathrm{M} \mathrm{NaCl}$ ( $n=$ 1.333-1.350). (C) Real-time sensorgram of $\mathrm{NaCl}$ injections and surface rinses with nanopure water. (D) Bulk refractive index sensitivities of gold-coated 3D printed, SF2, and BK7 prisms ( $n=3$ for all data points). 
A
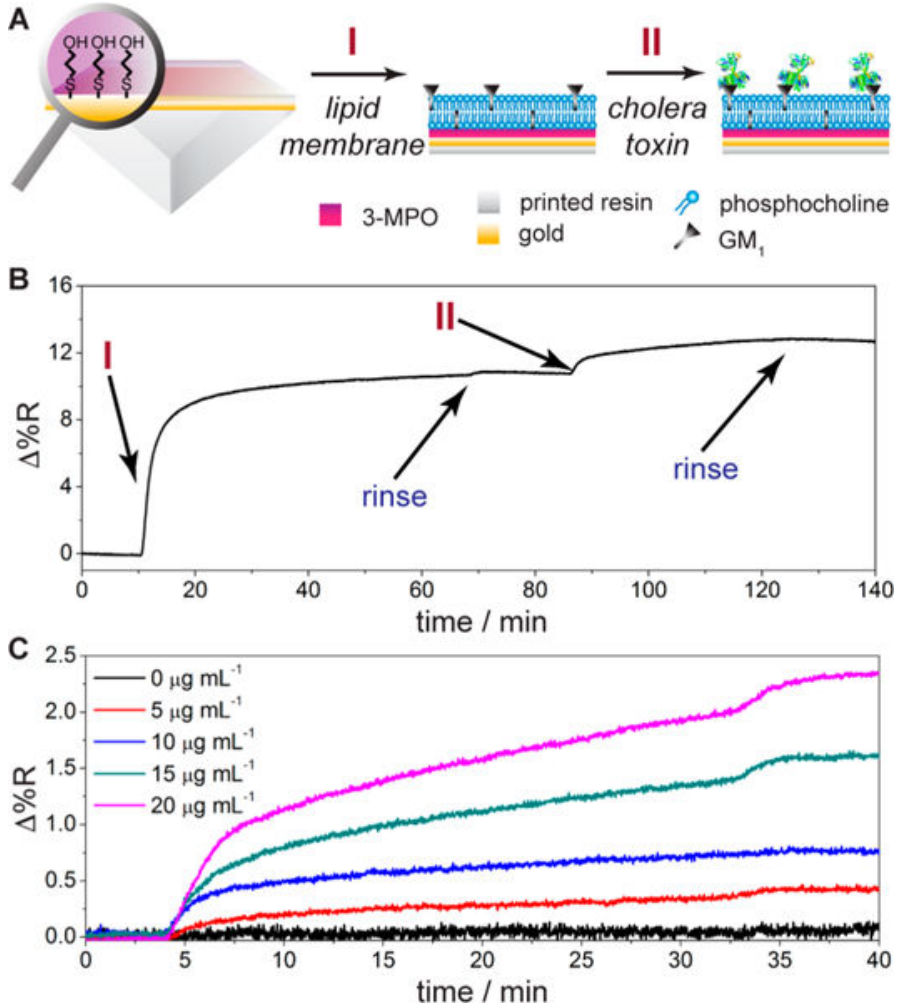

Figure 3.

Biosensing of cholera toxin. (A) Sensor fabrication and recognition scheme, utilizing of supported lipid membrane on 3-mercaptopropanol, followed by cholera toxin incubation. (B) SPR sensorgram of supported membrane formation and cholera toxin $\left(20 \mu \mathrm{g} \mathrm{mL}^{-1}\right)$ recognition. (C) Real-time binding of cholera toxin $\left(0-20 \mu \mathrm{gL}^{-1}\right)$ to the membraneembedded ganglioside receptor. 


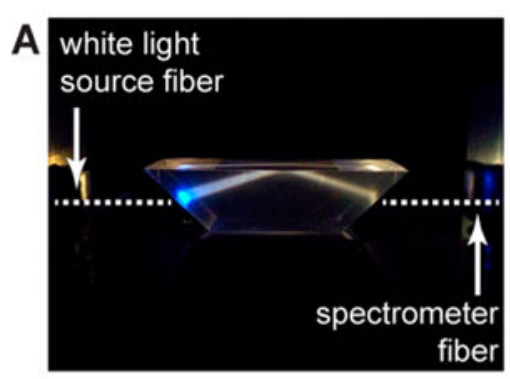

B
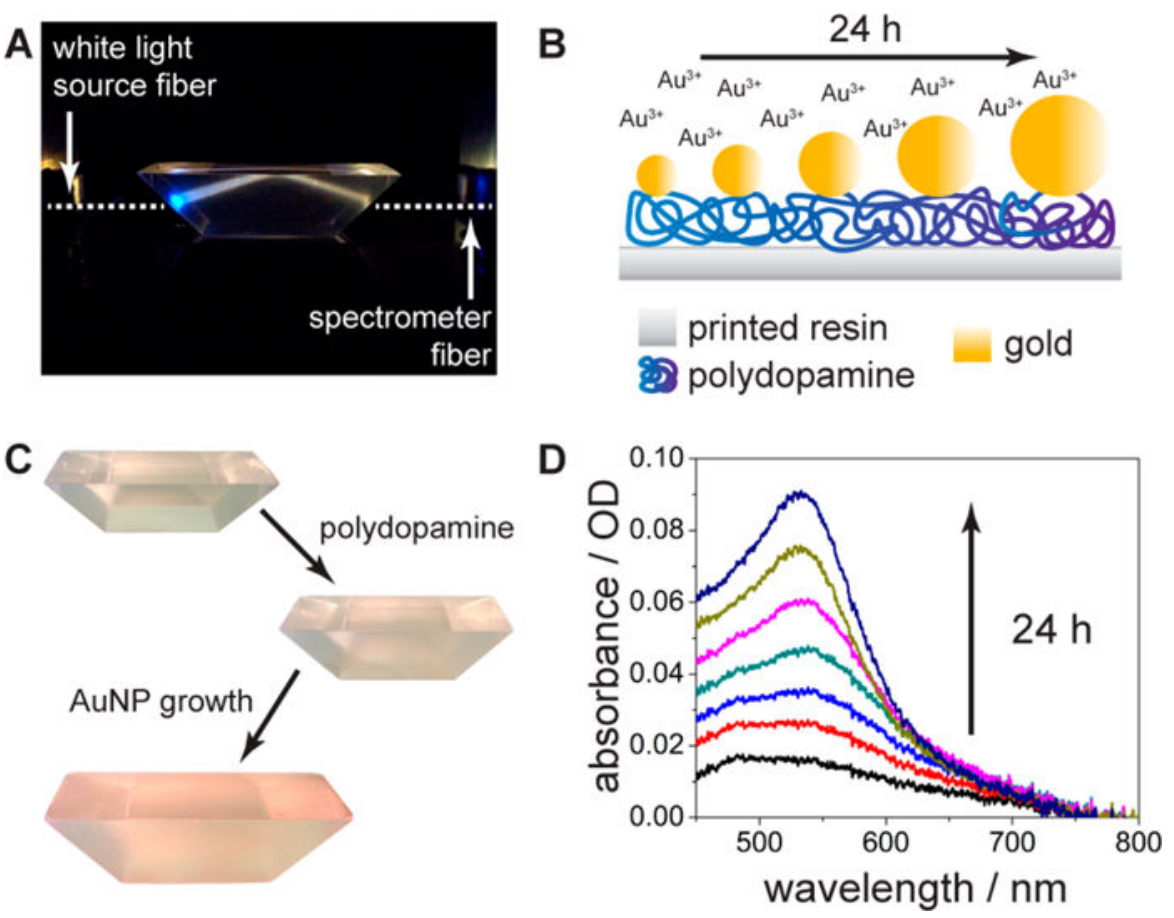

Figure 4.

Dove prism fabrication. (A) Absorbance setup with dove prism, with total internal reflection of the light path depicted. (B) Polydopamine-mediated nanoparticle growth schematic. (C) Photographs of dove prisms during various stages of nanoparticle functionalization. (D) Absorbance spectra of nanoparticle growth on the polydopamine functionalized dove prism surface over a $24 \mathrm{~h}$ period. 